\title{
Papulosis maligna atrophicans Köhlmeier-Degos ohne Systembefall bei einer 21-jährigen Patientin
}

\section{Malignant Atrophic Papulosis (Köhlmeier-Degos) Limited to the Skin} in a 21-Year Old Female Patient

\section{Autoren}

Institute

\author{
A. Holzer ${ }^{1}$, L. Cerroni², T. Haidn' ${ }^{1}$, R. R. Müllegger
}

Abteilung für Dermatologie, Landesklinikum Wiener Neustadt, Österreich

${ }^{2}$ Universitätsklinik für Dermatologie, Medizinische Universität Graz, Österreich

\section{Bibliografie}

Dol $10.1055 / \mathrm{s}-2007-995764$

Akt Dermatol 2008; 34:

124-127 @ Georg Thieme

Verlag KG Stuttgart · New York ISSN 0340-2541

\section{Korrespondenzadresse}

Dr. Anna Holzer

Abteilung für Dermatologie

Landesklinikum

Wiener Neustadt

Corvinusring 3-5

A-2700 Wiener Neustadt anna.holzer@wienerneustadt. Iknoe.at

\section{Zusammenfassung \\ $\nabla$}

Die Papulosis maligna atrophicans KöhlmeierDegos ist eine sehr seltene Erkrankung unbekannter Ätiologie. Sie ist durch disseminierte erythematöse Papeln, die mit atrophen porzellanweißen Narben und umgebendem teleangiektatischem Ring abheilen, an Stamm und Extremitäten charakterisiert. Bei einem Großteil der Patienten kommt es nach variabler Zeitdauer zu einem Systembefall, am häufigsten des Gastrointestinaltrakts und des Zentralnervensystems. Es wird über eine 21-jährige Patientin berichtet, bei der die Diagnose einer Papulosis maligna atrophicans anhand des distinkten klinischen Bildes

\section{Einleitung}

$\nabla$

Die Papulosis maligna atrophicans KöhlmeierDegos wurde erstmals 1941 vom Österreicher Köhlmeier als Variante der Thrombangitis obliterans beschrieben [1]. Ein Jahr später berichteten Degos et al. über einen Patienten mit analogen Hautveränderungen und nannten die Erkrankung „Dermatite papulo-squameuse atrophiante“ [2]. Seither sind in der Literatur erst etwa 200 Fälle dieser potenziell letalen Multisystemerkrankung unbekannter Ätiologie beschrieben worden. Sie tritt vor allem bei Kaukasiern auf und ist durch distinkte kutane Papeln an Stamm und Extremitäten charakterisiert.

In den 1980er Jahren wurden erstmals einzelne Fälle beschrieben, bei denen die Erkrankung auf die Haut limitiert blieb. Aufgrund der sehr variablen Zeitspanne zwischen dem Auftreten von Hautveränderungen und extrakutanen Manifestationen lässt sich allerdings bis heute nicht mit Sicherheit sagen, ob eine derartige benigne Form der Erkrankung existiert. Wir berichten über eine und der charakteristischen histopathologischen Veränderungen gestellt wurde. Sämtliche Laboruntersuchungen inklusive der Gerinnungs-, Autoimmun- und Vaskulitisparameter blieben ebenso ohne pathologischen Befund wie die direkte Immunfluoreszenz. In dem vierjährigen Krankheitsverlauf kam es zu keinen extrakutanen Manifestationen. Es existiert bislang keine sicher effektive Therapie. Bei unserer Patientin konnten weder Kortikosteroidexterna noch Pimecrolimus Creme die Hautveränderungen beeinflussen. Unsere Patientin könnte einen der seltenen Fälle einer benignen, auf die Haut limitierten Variante der Papulosis maligna atrophicans repräsentieren.

21-jährige Patientin mit 4-jährigem Bestehen von charakteristischen Hautveränderungen der Papulosis maligna atrophicans ohne Systembefall.

\section{Kasuistik \\ $\nabla$}

Anamnese

Bei der 21-jährigen Patientin traten im 18. Lebensjahr erstmals Hautveränderungen an den Beinen auf, die sich allmählich auf den Stamm und die oberen Extremitäten ausbreiteten. Die Patientin war bei subjektivem Wohlbefinden und gab keinerlei allgemeine Krankheitszeichen an. Grunderkrankungen oder Allergien waren nicht bekannt, es wurden keine Medikamente eingenommen. Wiederholt durchgeführte Therapien mit topischen Steroiden hatten zu keiner Verbesserung der Hautveränderungen geführt, die sich jeweils stadienhaft binnen einiger Wochen vom Zeitpunkt des ersten Auftretens umwandelten. 

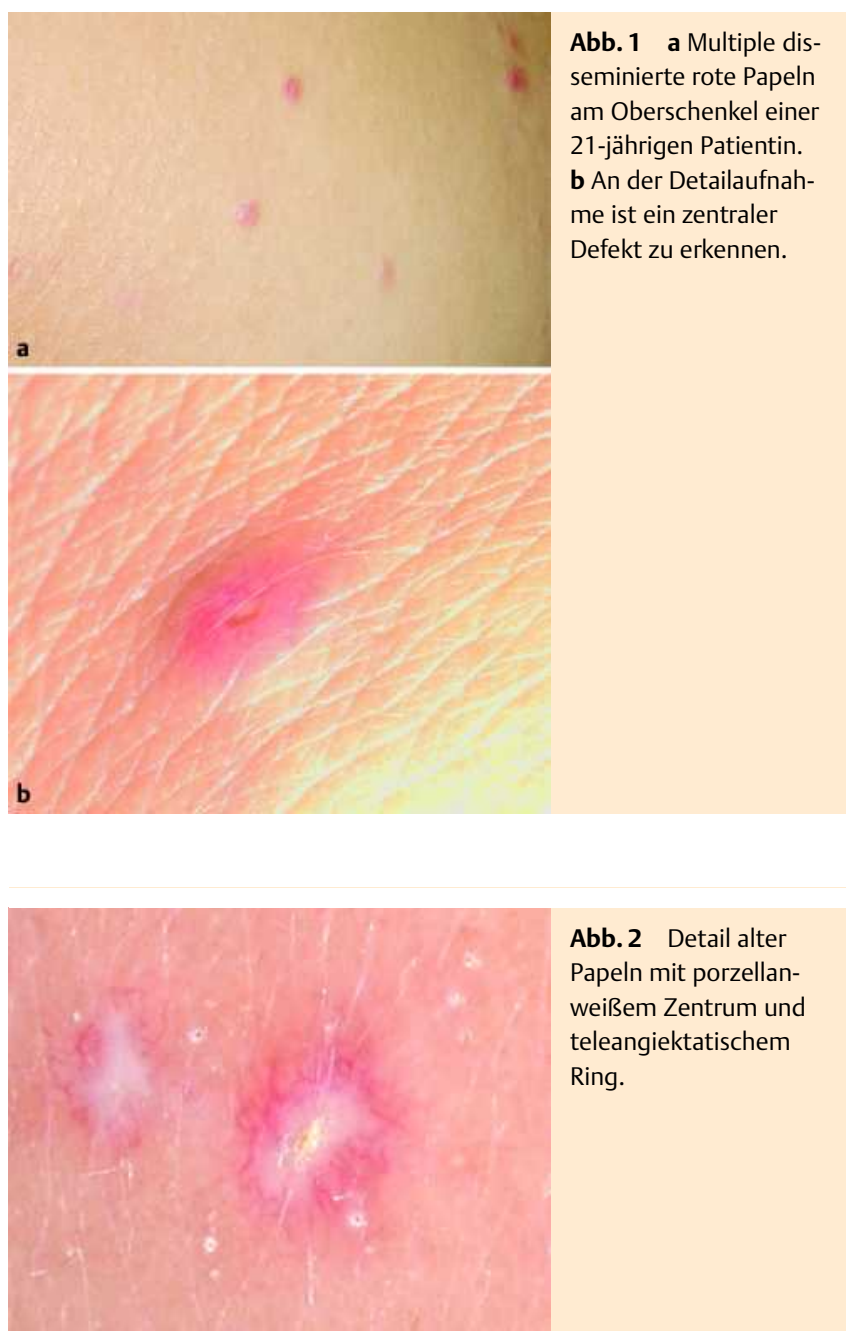

\section{Erstbefund}

Bei der klinischen Untersuchung fielen regellos disseminierte erythematöse Papeln bis $5 \mathrm{~mm}$ Größe an Stamm und Extremitäten auf. Etliche dieser Papeln zeigten eine zentrale Nabelung bzw. Erosion ( $\bullet$ Abb. 1). Andere imponierten mit zentralen porzellanweißen atrophen Närbchen und waren von einem teleangiektatischen Randsaum umgeben ( Abb. 2).

\section{Differenzialdiagnosen}

Aufgrund des distinkten klinischen Bildes zogen wir primär Atrophie blanche-artige Papeln bei Lupus erythematodes bzw. Dermatomyositis oder eine Papulosis maligna atrophicans in Betracht. Andere papulöse Hauterkrankungen inklusive lymphomatoide Papulose, Lichen planus, papulöses Granluoma anulare, kleinknotige Sarkoidose, multiple persistierende Arthropodenreaktionen, Prurigo simplex chronica und multiple Dermatofibrome betrachteten wir aufgrund von Anamnese, Verteilungsmusters und/oder Morphe als wenig wahrscheinlich.

\section{Befunde diagnostischer Untersuchungen}

Die histopathologische Untersuchung einer Papel zeigte neben einer Atrophie der Epidermis ausgeprägte Muzinablagerungen in der Dermis (Spezialfärbung mit Alzianblau), eine Degeneration kleiner Blutgefäße mit fibrinoiden Thromben und Endothel-

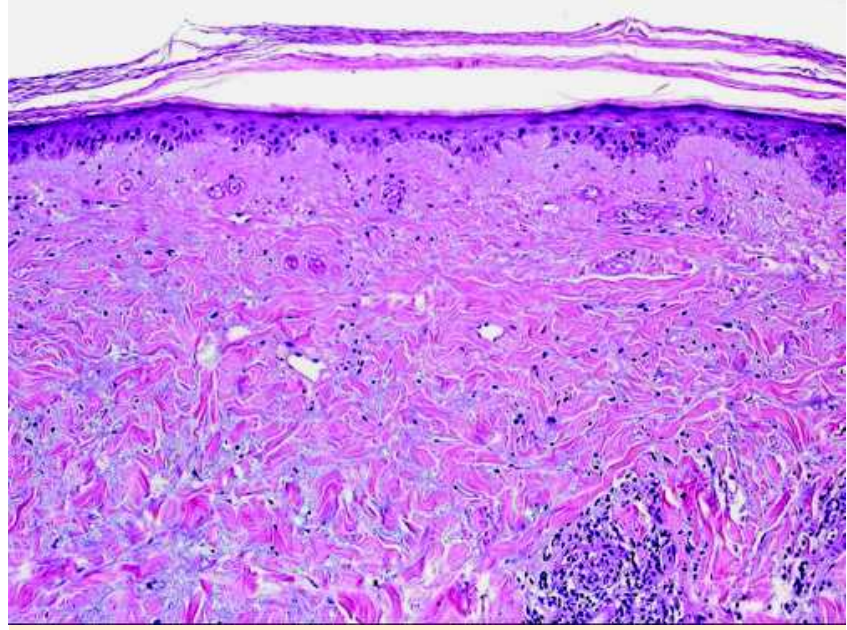

Abb. 3 Die histopathologische Untersuchung einer Papel zeigt unter einer atrophen Epidermis durch fibrinoide Thromben verschlossene kleine Blutgefäße, ein perivaskuläres lymphohistiozytäres Infiltrat und ausgeprägte Muzinablagerungen (Hämatoxylin und Eosin, $\times 40$ ).

zellschwellungen sowie ein perivaskuläres lymphohistiozytäres Infiltrat $($ Abb.3). Die direkte Immunfluoreszenzuntersuchung periläsioneller Haut zeigte keine spezifischen Ablagerungen von Immunreaktanzien.

Klinische Chemie, komplettes Blutbild und großer Gerinnungsstatus waren unauffällig. ANA, ANCA, die Komplementfaktoren C3 und C4, zirkulierende Immunkomplexe, Antiphospholipidantikörper sowie ACE waren durchwegs im Normbereich. Hepatitis- und HIV-Antikörper waren negativ.

In Synopsis von klinischem Bild und Dermatohistopathologie stellten wir die Diagnose einer Papulosis maligna atrophicans Köhlmeier-Degos.

\section{Ergänzende Untersuchungen}

Die weitere Abklärung ergab jeweils unauffällige Ergebnisse von Elektrokardiografie, Thorax Röntgen, kranialer Magnetresonanztomografie und -angiografie sowie Computertomografie und Sonografie des Abdomens. In der Gastroskopie wurde ein MalloryWeiß Riss und eine petechiale Antrumgastritis gefunden, die histopathologische Untersuchung ergab keinen Hinweis für eine Manifestation der Papulosis maligna atrophicans. Bei der Koloskopie mit Stufenbiopsie zeigte sich eine unspezifische Entzündung in Sigma, Rektum und Ileum mit lymphoider Hyperplasie.

\section{Therapie und Verlauf}

Aufgrund des benignen Verlaufs der Erkrankung, des Fehlens einer sicher effektiven Therapie und weil die Patientin einer Behandlung ablehnend gegenüberstand, wurde kein Versuch einer systemischen Medikation unternommen. Ein Jahr nach Erstvorstellung und somit nach einer Gesamtkrankheitsdauer von vier Jahren ist es bei unserer Patientin zu keinem Systembefall gekommen. Die topische Applikation von Methylprednisolon aceponat und von Pimecrolimus $1 \%$ Creme in einem Halbseitenversuch über 4 Wochen zeigte keinen Effekt. Das Hautzustandsbild blieb stabil bzw. kam es über das 1-Jahres Follow-up nur zu einer geringen Zunahme der Zahl an Papeln. 


\section{Diskussion \\ $\nabla$}

Die Papulosis maligna atrophicans Köhlmeier-Degos, im angloamerikanischen Sprachraum ist die Bezeichnung „Degos' disease“ gebräuchlich, ist eine sehr seltene Erkrankung unbekannter Ätiologie [3]. Elektronenmikroskopische Befunde tubulärer Strukturen in Endothelzellen betroffener Gefäße haben an eine Paramyxovirusinfektion als Auslöser denken lassen [4]. Da die Papulosis maligna atrophicans einige histologische Merkmale eines Lupus erythematodes aufweist (vor allem Muzinablagerung, gelegentlich auch eine Interphase-Dermatitis), wurde manchmal eine Assoziation zu dieser Autoimmunkrankheit postuliert [5]. Andere Autoren favorisieren eine primäre Koagulopathie [6,7] oder eine Dysfunktion der Endothelzellen [8] als primäre Ursache der Erkrankung. Obwohl definitive Beweise für all diese Hypothesen bis heute ausstehen, wird die Papulosis maligna atrophicans in modernen dermatologischen Büchern als mikrovaskulär-okklusive Krankheit klassifiziert. Entsprechende (Labor)untersuchungen haben bei unserer Patientin keinen Hinweis für eine Assoziation zu einer der diskutierten Ursachen gegeben. Die pathogenetische Kaskade scheint mit einer Endothelzellaffektion zu beginnen, die von lokalen Thrombosen gefolgt wird, was konsekutiv Infarzierungen in den betroffenen Gewebsabschnitten nach sich zieht [5].

An der Haut manifestiert sich die Erkrankung mit schütter disseminierten erythematösen Papeln, vor allem an Stamm und Extremitäten. Diese Papeln heilen nach einigen Wochen unter Hinterlassung charakteristischer porzellanweißer atropher Närbchen mit einem teleangiektatischen Randsaum ab. Neben diesem typischen klinischen Bild ist die Histopathologie entscheidend für die Diagnose. Während frühe Läsionen durch ein superfizielles und tiefes perivaskuläres lymphohistiozytäres Infiltrat, vakuoläre Interface Dermatitis und interstitielle Muzinablagerungen gekennzeichnet sind, sieht man bei älteren Läsionen eine Atrophie der Epidermis, degenerative Gefäßveränderungen (gelegentlich mit Mikrothromben), keilförmige Sklerose und ein spärliches lymphozytäres Infiltrat in der oberen Dermis [9]. Wegweisende Laborparameter existieren nicht.

Die Bezeichnung der Erkrankung als maligne resultiert aus dem Umstand, dass es bei ungefähr 85\% der Patienten zu einer extrakutanen Systembeteiligung kommt. Am häufigsten sind der Gastrointestinaltrakt (60\%) und das Zentralnervensystem (30\%) betroffen. Dünndarmperforation mit konsekutiver Peritonitis ist die schwerste Komplikation und die häufigste Todesursache. Weitere wiederholt beschriebene Komplikationen sind zerebrale Blutung, Perikarditis, Pleuritis, Polyneuropathie und ophthalmologische Manifestationen [3]. Die Gesamtletalität bei Papulosis maligna atrophicans mit Systembefall liegt bei zirka $60 \%$, die meisten Patienten versterben innerhalb von zwei Jahren nach Auftreten der ersten Systemmanifestationen [10]. Derzeit geht man davon aus, dass es neben dieser malignen Form der Papulosis maligna atrophicans mit Systembeteiligung und hoher Mortalität auch eine benigne, rein kutane Variante gibt. Benign verlaufende Fälle wurden vor allem in den letzten Jahren beschrieben [11-13]. Hierzu muss jedoch kritisch angemerkt werden, dass aus der Literatur bekannt ist, dass sich extrakutane Manifestationen nach sehr variabler Zeit (einigen Monaten bis zu zehn Jahren) einstellen können [3,14]. Das unterschiedlich lange Follow-up dieser Patienten war jedoch möglicherweise nicht ausreichend, um tatsächlich die Existenz einer benignen Variante der Papulosis maligna atrophicans zu bestätigen. Prognostische Marker für das Auftreten einer Systembeteiligung bzw. für die Differenzierung der beiden Varianten fehlen. Deshalb sind bei Patienten mit Papulosis maligna atrophicans in jedem Fall regelmäßig Langzeitkontrollen angezeigt.

Bislang ist keine eindeutig effektive Therapie für die Papulosis maligna atrophicans etabliert. In einzelnen Fällen konnten Patienten, die keine Systembeteiligung aufwiesen, erfolgreich mit Thrombozytenaggregationshemmern wie zum Beispiel Acetylsalicylsäure behandelt werden, teils auch in Kombination mit Pentoxifyllin [9]. In einem anderen Fall heilten die Hautveränderungen nach mehrwöchiger Applikation eines transdermalen Nikotinpflasters ab [15]. Eine Therapie mit Immunsuppressiva wie Glukokortikoiden, Methotrexat, Cyclosporin A, Azathioprin oder Cyclophosphamid, sowie zahlreiche andere Therapieversuche einschließlich Heparin, Östrogenen und Antibiotika, haben sich als ineffektiv erwiesen. Im Falle diverser extrakutaner Komplikationen muss eine entsprechende symptomatische Therapie durchgeführt werden. Bei unserer Patientin wurde keine Systemtherapie durchgeführt, weil keine extrakutanen Krankheitsmanifestationen bestanden und die Patientin eine solche ablehnte. Weder topische Kortikosteroide noch Pimecrolimus konnten die Hautveränderungen beeinflussen. Bei unserer Patientin blieb die Erkrankung bislang über einen Zeitraum von vier Jahren auf die Haut beschränkt, wo sie eine nur sehr geringgradige Zunahme von Papeln zeigte. Nachdem die durchschnittliche Periode zwischen dem Auftreten von Hautveränderungen und extrakutanen Manifestationen in der Literatur mit etwa 2 Jahren beschrieben ist, scheint unsere Patientin den Fall einer benignen Variante der Papulosis maligna atrophicans zu repräsentieren.

Zusammenfassend beschreiben wir einen der sehr seltenen Fälle einer jahrelang auf die Haut limitierten Papulosis maligna atrophicans bei einer jungen Frau („benigne Variante“). Neben dem distinkten klinischen Bild mit regellos disseminierten erythematösen Papeln mit konsekutiver Ausbildung atropher zentraler porzellanweißer Narben und teleangiektatischem Randsaum war die Histopathologie diagnostisch wegweisend. Aufgrund des hohen Risikos für die Entwicklung (schwerwiegender) extrakutaner Komplikationen ist ein konsequentes Langzeit-Followup aller Patienten indiziert.

\section{Abstract}

\section{Malignant Atrophic Papulosis (Köhlmeier-Degos) Limited to the Skin in a 21-Year Old Female Patient $\nabla$}

Malignant atrophic papulosis Köhlmeier-Degos is a very rare disease of unknown etiology. It affects the skin with disseminated erythematous papules on the trunk and extremities, which heal with atrophic porcelain-white scars with a telangiectatic rim. In the majority of patients, extracutaneous manifestations, mostly of the gastrointestinal tract or the central nervous system, develop after a variable period of time. We report a 21 -yearold female patient in whom the diagnosis of malignant atrophic papulosis was based on the distinct clinical appearance and histopathologic alterations. Laboratory investigations, including haemostasis, autoimmunity, and vasculitis specific parameters were all negative, as was direct immunfluorescence. The patient has had no extracutaneous manifestations during a 4-yeardisease duration. There is no established effective treatment. Neither topical corticosteroids nor pimecrolimus were of any benefit in our patient. This patient may represent one of the rare 
cases of a benign variant of malignant atrophic papulosis limited to the skin.

\section{Literatur}

1 Köhlmeier W. Multiple Hautnekrosen bei Thrombangitis obliterans. Arch Dermatol Syph 1941; 181: 783-792

2 Degos $R$, Delort J, Tricot $R$ et al. Dermatite papulosquameuse atrophiante. Bull Soc FR Derm Syph 1942; 49: 48 - 150

3 Scheinfeld N. Malignant atrophic papulosis. Clin Exp Dermatol 2007; 32: $483-487$

4 Howard RO, Nishida S. A case of Degos' disease with electron microscopic findings. Transact Am Acad Ophthalmol Otolaryngol 1969; 73: $1097-1112$

5 Ball E, Newburger A, Ackermann AB. Degos' disease: A distinctive pattern of disease, chiefly of Lupus erythematosus, and not a specific disease per se. Am Journal Dermatopathol 2003; 25: 308 - 320

6 Paramo JA, Rocha E, Cuesta B, Rocha Hernando E et al. Fibrinolysis in Degos' disease. Thromb Haemost 1985; 54: 730

7 Vazquez-Doval FJ, Ruiz de Erenchun F, Paramo JA, Quintanilla E. Malignant atrophic papulosis: A report of two cases with altered fibrinolysis and platelet function. Clin Exp Dermatol 1993; 18: 441 - 444

8 Tribble K, Archer ME, Jorizzo JL, Cavallo T et al. Malignant atrophic papulosis: Absence of circulating immuncomplexes or vasculitis. J Am Acad Dermatol 1986; 15: 365 - 369

9 Harvell JD, Williford PL, White WL. Benign cutaneous Degos' disease: a case report with emphasis on histopathology as papules chronologically evolve. Am J Dermatopathol 2001; 23: 116-123

10 Viktor C, Schultz-Ehrenburg U. Malignant atrophic papulosis (Köhlmeier-Degos): diagnosis, therapy and course. Hautarzt 2001; 52: $734-$ 737

11 Wilson J, Walling HW, Stone MS. Benign cutaneous Degos Disease in a 16-year-old girl. Pediatr Dermatol 2007; 24: 18 - 24

12 Loewe R, Palatin M, Petzelbauer P. Degos' disease with an inconspicuous clinical course. JEADV 2005; 19: 477-480

13 Zamiri $M$, Jarrett $P$, Snow J. Benign cutaneous Degos disease. Int J Dermatol 2005; 44: 654-656

14 Plantin P, Labouche F, Sassolas B, Delaire P, Guillet G. Degos' disease: a 10 -year follow-up of a patient without visceral involvement. J Am Acad Dermatol 1989; 21: 136-137

15 Kanekura T, Uchino Y, Kanzaki T. A case of malignant atrophic papulosis successfully treated with nicotine patches. Br J Dermatol 2003; 149: $660-662$

\section{Preisverleihung}

Verleihung des 9. Arnold Rikli-Preises beim Symposium "Licht und Gesundheit", 13. März 2008 in Berlin

Die gemeinnützige Jörg Wolff-Stiftung in Stuttgart verfolgt unter anderem den Zweck, die Forschung und Lehre über die gesundheitlichen Wirkungen von natürlicher oder künstlich erzeugter optischer Strahlung auf den Menschen zu fördern. Die Tradition des erstmalig 1989 vergebenen Arnold Rikli-Preises wurde 2006 von der Jörg Wolff-Stiftung übernommen, die seitdem jährlich diesen Forschungspreis ausschreibt. Mit dem Preis werden aktuelle Forschungsarbeiten ausgezeichnet, die biologische Wirkungen von ultravioletter, sichtbarer und infraroter Strahlung betreffen und direkte Bedeutung für die Humanmedizin haben oder Richtlinien für zukünftige Forschung liefern. Eine unabhängige wissenschaftliche Jury wählt aus den jeweiligen Bewerbungen den jährlichen Preisträger aus. Die Auszeichnung ist mit einem Preisgeld in Höhe von $10000 €$ verbunden.

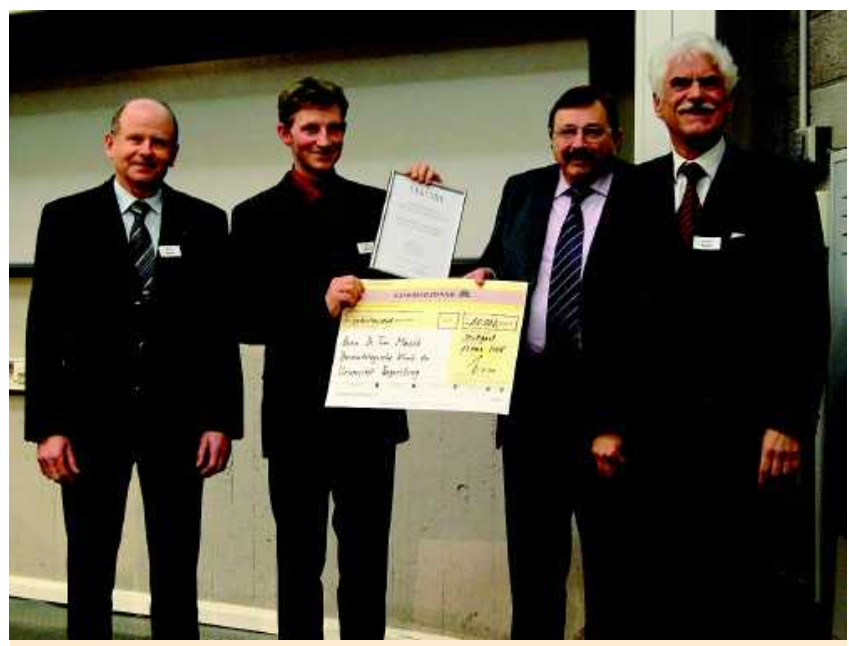

Von links nach rechts: Herr Prof. Hölzle (Laudator), der Preisträger Herr Dr. Maisch, Herr Dr. Bocionek (Vertreter der Jörg Wolff-Stiftung) und Herr Prof. Kaase (fachliche Gesamtleitung des Symposiums „Licht und Gesundheit“).

Die Vergabe des 9. Arnold Rikli-Preises erfolgte anlässlich des 6. Symposiums „Licht und Gesundheit“ am 13. März 2008 in Berlin. Vor der Übergabe der Urkunde an Herrn Dr. Tim Maisch von der Dermatologischen Klinik der Universität Regensburg würdigte Herr Professor Erhard Hölzle, Oldenburg, als Sprecher der Jury in seiner Laudatio den Preisträger für das Jahr 2007 und die Bedeutung seiner Forschungstätigkeiten. Die ausgezeichnete Arbeit über die Rolle von Singulett-Sauerstoff und der Sauerstoffkonzentration bei der photodynamischen Inaktivierung von Bakterien stellte im Anschluss daran der Preisträger selbst vor. 